\title{
Influencia del tiempo interpaso, la deformación y la temperatura final sobre la tensión acumulada en la austenita para un acero microaleado al vanadio
}

\author{
M. Gómez*, S.F. Medina*y A. Quispe* \\ Resumen La temperatura de no-recristalización $\left(T_{\mathrm{nr}}\right)$ y la temperatura de transformación de fase \\ durante el enfriamiento $\left(A_{\mathrm{r} 3}\right)$ de un acero microaleado al vanadio de medio carbono han sido \\ determinadas mediante simulación de laminación en caliente bajo diferentes condiciones \\ de deformación aplicada y tiempo interpaso. Sobre la curva MFS se ha determinado la \\ tensión acumulada en la austenita $(\Delta \sigma)$ a temperaturas comprendidas entre $T_{\mathrm{nr}}$ y $A_{\mathrm{r} 3}$ y se \\ ha estudiado la influencia de las condiciones de deformación sobre el valor de $T_{\mathrm{nr}}$ y de $\Delta \sigma$. \\ Las temperaturas $T_{\mathrm{nr}}$ y $A_{\mathrm{r} 3}$ establecen el intervalo donde deberían efectuarse los últimos \\ pasos de la laminación industrial, con vistas a obtener una austenita endurecida que \\ conducirá a una microestructura final más fina.
}

Palabras clave Acero microaleado. Simulación de laminación en caliente. Temperaturas críticas de laminación. Tensión acumulada. Influencia de las condiciones de laminación.

\section{Influence of interpass time, strain and final temperature on austenite accumulated stress for a vanadium microalloyed steel}

\begin{abstract}
Non-recrystallization temperature $\left(T_{\mathrm{nr}}\right)$ and phase transformation temperature during cooling $\left(A_{\mathrm{r} 3}\right)$ of a medium carbon, vanadium-microalloyed steel have been determined by means of hot rolling simulation under different conditions of strain applied and interpass time. The stress accumulated in the austenite at temperatures between $T_{\mathrm{nr}}$ and $A_{\mathrm{r} 3}(\Delta \sigma)$ has been measured on MFS curve and the influence of the deformation conditions on $T_{\mathrm{nr}}$ and $\Delta \sigma$ values has been studied. $T_{\mathrm{nr}}$ and $\mathrm{A}_{\mathrm{r} 3}$ are the temperature limits where last passes of industrial rolling should be accomplished in order to obtain a strengthened austenite leading to a finer final microstructure.
\end{abstract}

Keywords Microalloyed steel. Hot rolling simulation. Rolling critical temperatures. Accumulated stress. Influence of rolling conditions.

\section{INTRODUCCIÓN}

La temperatura de no-recristalización, $T_{n r}$, y las temperaturas de transformación en enfriamiento, $A_{\mathrm{r} 3}$ y $A_{\mathrm{r} 1}$, deben considerarse las temperaturas críticas de la laminación en caliente del acero. $T_{\mathrm{nr}}$ es la temperatura por debajo de la cual la recristalización estática de la austenita comienza a ser inhibida durante la laminación y, es un parámetro de gran importancia por su influencia en la microestructura austenítica al final de la laminación, que podrá ser totalmente recristalizada o endurecida, en mayor o menor grado. Las temperaturas de transformación de fase, $A_{\mathrm{r} 3}$ y $A_{\mathrm{r} 1}$, son asimismo importantes, pues sus valores indican los límites de temperatura entre los cuales la laminación ha de finalizar, bien en la región austenítica o en la región intercrítica. 
Influencia del tiempo interpaso, la deformación y la temperatura final sobre la tensión acumulada en la austenita para un acero microaleado al vanadio

M. GÓMEZ, S.F. MEDINA y A. QUisPE

El parámetro $T_{\mathrm{nr}}$ puede determinarse mediante el método de Jonas et al., consistente en la simulación de sucesivos pasos de laminación y posterior representación gráfica de la tensión media de fluencia (MFS) frente a la inversa de la temperatura para cada paso ${ }^{[1]}$. El valor de $T_{\text {nr }}$ depende de la composición química del acero, la deformación equivalente aplicada en cada paso de la laminación $(\varepsilon)$, la velocidad de deformación $(\dot{\varepsilon})$ y el tiempo interpaso $(\Delta t)^{[1}$ y 2$]$.

Por su parte, los valores de $A_{r 3}$ y $A_{r 1}$ dependen de la composición química, de la velocidad de enfriamiento y de la microestructura austenítica (tamaño de grano y endurecimiento) antes de la transformación de fase $\gamma \rightarrow \alpha . A_{\mathrm{r} 3}$ y $A_{\mathrm{r} 1}$ pueden hallarse por métodos clásicos y de gran precisión como la dilatometría o simultáneamente a $T_{\mathrm{nr}}$, usando el método de la MFS [2 y 3]. No obstante, la determinación de las temperaturas de transformación de fase mediante esta segunda técnica es mucho más sencilla en aceros con muy bajo contenido en carbono ${ }^{[4]}$.

Además de estas temperaturas críticas, sobre la curva MFS también puede determinarse gráficamente la tensión residual o acumulada en la austenita $(\Delta \sigma)$, que es una magnitud que representa el progresivo endurecimiento de la austenita a medida que ésta va siendo deformada a temperaturas inferiores a $T_{\mathrm{nr}} . \Delta \sigma$ supone una magnitud apropiada para relacionar la microestructura de la austenita antes del comienzo de la transformación $\gamma \rightarrow \alpha$ con el tamaño de grano ferrítico final ${ }^{[5]}$, que es la magnitud más influyente en las propiedades mecánicas finales del acero ${ }^{[6]}$.

Se expone en el presente trabajo un método grafico, rápido y directo, para determinar simultáneamente las temperaturas críticas de laminación y la tensión acumulada. Se avanza en este último concepto respecto a interpretaciones previas que sólo consideraban la tensión acumulada en los instantes previos a la transformación ${ }^{[3}$ y 7], empleándose ahora $\Delta \sigma$ como medio de caracterización del endurecimiento de la austenita durante todo el intervalo de temperaturas $\left(T_{\mathrm{nr}}-\mathrm{A}_{\mathrm{r} 3}\right)$.

\section{MATERIALES Y PROCEDIMIENTO EXPERIMEN- TAL}

El acero estudiado, cuya composición química se muestra en la tabla I, es un acero con un contenido medio-alto de carbono y un alto contenido de vanadio y nitrógeno, fabricado por refusión bajo electroescoria.

La simulación termomecánica se llevó a cabo por medio de ensayos de torsión en caliente de múltiples pasos. Las probetas, con un diámetro de $6 \mathrm{~mm}$ y una longitud útil de $50 \mathrm{~mm}$, fueron austenizadas a $1.200{ }^{\circ} \mathrm{C}$ durante $10 \mathrm{~min}$. Estas condiciones fueron suficientes para disolver completamente los precipitados de vanadio, ya que las temperaturas de solubilidad calculadas para nitruros y carburos fueron respectivamente de $1.141{ }^{\circ} \mathrm{C}$ y $904{ }^{\circ} \mathrm{C}{ }^{[8]}$. La temperatura fue, entonces, rápidamente reducida hasta la correspondiente al primer paso $\left(1.150^{\circ} \mathrm{C}\right)$, estableciéndose una velocidad de enfriamiento de $25^{\circ} \mathrm{C}$ entre pasos sucesivos hasta alcanzar el paso final a $650^{\circ} \mathrm{C}$. Se realizaron diversas simulaciones para diferentes valores de $\Delta \mathrm{t}(20$, $30,60,100,200$ y $500 \mathrm{~s}$ ) y dos deformaciones de 0,20 y 0,35 . La velocidad de deformación fue la misma para todos los casos $\left(\dot{\varepsilon}=3,63 \mathrm{~s}^{-1}\right)$.

\section{RESULTADOS Y DISCUSIÓN}

En el ensayo de torsión se obtienen los valores del par aplicado frente al número de giros realizados sobre la probeta, que son transformados respectivamente a tensión y deformación equivalentes mediante el criterio de Von Mises ${ }^{[9]}$. De este modo, es posible trazar las curvas tensión-deformación y calcular MFS para cada paso. El valor de MFS se determina calculando, por integración numérica, el área bajo la curva tensión-deformación correspondiente a cada paso y dividiendo este valor por la deformación aplicada. Si se representa gráficamente MFS frente a 10.000/T se obtienen curvas como la de la figura 1 , donde es posible determinar las temperaturas críticas y la tensión acumulada en la austenita. El comienzo del endurecimiento de la

Tabla I. Composición química del acero estudiado (\% contenido en masa)

Table I. Chemical composition of steel studied (mass \%)

\begin{tabular}{|c|c|c|c|c|c|c|c|c|c|c|}
\hline C & $\mathrm{Si}$ & $M n$ & $\mathbf{P}$ & $S$ & Al & $\mathrm{Cu}$ & $\mathrm{Cr}$ & v & $\mathbf{N}$ & 0 \\
\hline 0,48 & 0,28 & 1,45 & 0,024 & 0,018 & 0,009 & 0,008 & 0,22 & 0,13 & 0,0200 & 0,0044 \\
\hline
\end{tabular}




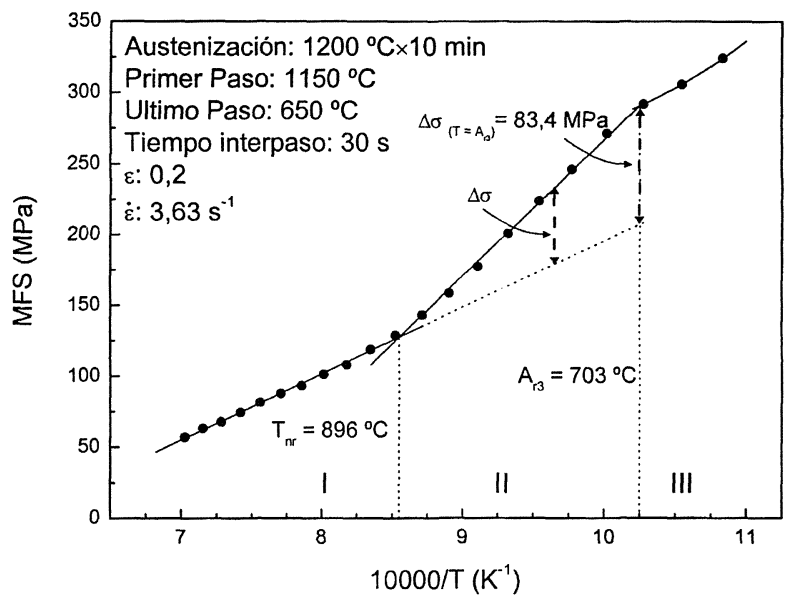

Figura 1. Tensión media de fluencia (MFS) frente a la inversa de la temperatura absoluta. Temperaturas críticas y tensión acumulada en la austenita a $T_{\mathrm{nr}}>\mathrm{T}>\mathrm{A}_{\mathrm{r} 3}$.

Figure 1. Mean flow stress (MFS) versus inverse of the absolute temperature. Critical temperatures and stress accumulated in the austenite at $T_{n r}>T>A_{r 3}$.

austenita, marcado por $T_{\mathrm{nr}}$, se define por el punto de intersección de las dos rectas de regresión, mientras que el inicio de la formación de ferrita, establecido por $A_{r 3}$, se localiza en el punto a bajas temperaturas donde cambia, de nuevo, la pendiente. La determinación de las temperaturas de transformación de fase es mucho más sencilla para aceros con bajo contenido de carbono, donde el alto porcentaje de ferrita formado provoca un marcado descenso en el valor de la MFS. Para estos aceros, la formación de un tramo final de la curva MFS asimilable a una parábola permite distinguir con claridad las temperaturas $A_{\mathrm{r} 3}$ y $A_{\mathrm{r} 1}{ }^{[10]}$. Sin embargo, para el acero estudiado (con un contenido en carbono mayor a $0,45 \%$ ) la baja proporción de ferrita formada conlleva, tan sólo, un tenue descenso en la pendiente de la curva MFS que hace que la determinación de $A_{\mathrm{r} 3}$ sea, en ocasiones, complicada y que resulte imposible hallar $A_{\mathrm{r} 1}$.

De acuerdo con lo anterior, es posible distinguir tres zonas con diferentes microestructuras, que han sido marcadas sobre la figura 1 :

- Zona I: corresponde a austenita que recristaliza completamente entre pasos.

- Zona II: corresponde a austenita deformada a $\mathrm{T}<T_{\mathrm{nr}}$, cuya recristalización se ve parcial o totalmente inhibida y que, por consiguiente, va acumulando un endurecimiento.

- Zona III: corresponde a las sucesivas transformaciones austenita-ferrita y austenita-perlita.

Cuando el tiempo interpaso es muy corto, el único mecanismo de retraso de la recristalización es el de la fricción ejercida por los solutos sobre los límites de grano. Para tiempos más largos, la inhibición de la recristalización a lo largo de la zona II tiene su origen en la precipitación inducida por la deformación de finas partículas de nitruros y carburos del elemento microaleante capaces de bloquear el avance del límite de grano austenítico. Esta inhibición produce un endurecimiento gradual o acumulación de tensión en la austenita, manifestada por el aumento de pendiente de la recta de regresión. Tal y como muestra la figura 1 , la cuantía de la tensión acumulada en la austenita $(\Delta \sigma)$ puede calcularse para cualquier temperatura de deformación comprendida entre $T_{\mathrm{nr}}$ y $A_{\mathrm{r} 3}$, midiendo la distancia vertical entre las dos rectas de regresión, alcanzándose el valor máximo en los instantes previos al comienzo de la transformación $\gamma \rightarrow \alpha$ $\left(\mathrm{T}=A_{\mathrm{r} 3}\right)$. Este método considera que, si la austenita recristalizase completamente entre pasos hasta alcanzar $A_{\mathrm{r} 3}$ no existiría $T_{\mathrm{n}}$, por lo que sólo habría una línea de regresión y $\Delta \sigma$ tomaría un valor nulo, incluso en $A_{\mathrm{r} 3}$. Este caso no se ha presentado para las condiciones de deformación empleadas, de modo que en los doce ensayos realizados se pudo determinar un valor de $T_{\mathrm{nr}}$, y $\Delta \sigma$ en $\mathrm{A}_{\mathrm{r} 3}$ tomó un valor mayor que 0 . La figura 2 muestra el valor de $\Delta \sigma$ en función de la temperatura final de laminación para las diferentes condiciones de deformación $(\varepsilon, \Delta \mathrm{t})$.

Los valores de $T_{\mathrm{nr}}, A_{\mathrm{r} 3}$ y $\Delta \sigma$ máxima, medidos sobre las curvas de MFS frente a $1 / T$ para las diferentes condiciones de simulación, se muestran en la tabla II, donde puede apreciarse como $T_{\mathrm{nr}}$ y $\Delta \sigma$ decrecen a medida que $\Delta t$ aumenta. Un incremento de $\Delta$ t significa una mayor posibilidad de que la austenita recristalice completamente entre pasos. Por otra parte, los valores de $T_{\mathrm{nr}}$ y $\Delta \sigma$, para un mismo valor de $\Delta t$, fueron menores cuando el valor de $\varepsilon$ fue mayor $(0,35)$ ya que el incremento en la deformación acelera la cinética de la recristalización estática entre pasos. Estos resultados indican que para alcanzar un considerable endurecimiento de la austenita durante la laminación, próximo al pancaking, es necesario aplicar bajas deformaciones y reducir el tiempo interpaso, tal y como ocurriría en el tren acabador. La cantidad de deformación y la temperatura son, junto con el tiempo transcurrido tras la deformación, las variables que afectan en mayor medida la cinética de la recristalización estática ${ }^{[11}$ y 12$]$. Aunque $\varepsilon$ y $\Delta t$ influyen de modo similar sobre la cinética de la precipitación capaz de inhibir la recristalización, esta dependencia es menos acusada que la de la 
Influencia del tiempo interpaso, la deformación y la temperatura final sobre la tensión acumulada en la austenita para un acero microaleado al vanadio

M. GÓMEZ, S.F. MEDINA Y A. QUISPE

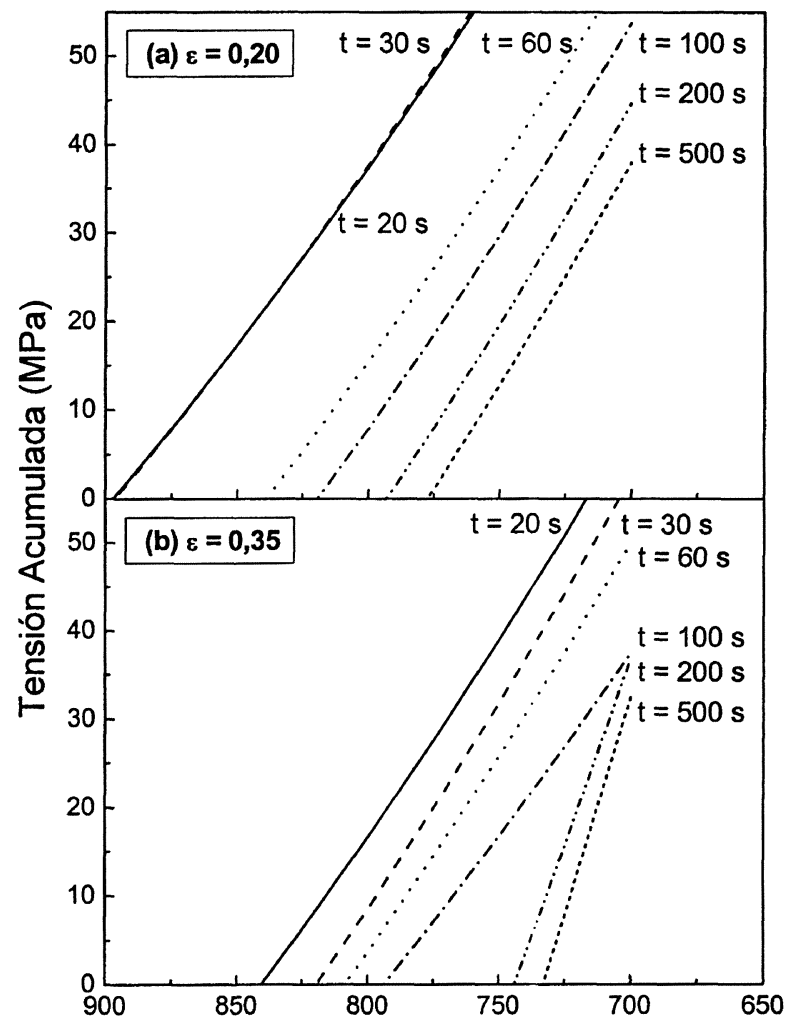

Figura 2. Evolución de la tensión acumulada en la austenita entre $T_{\mathrm{nr}}$ y $A_{\mathrm{r} 3}(\Delta \sigma)$ frente a la temperatura final de laminación. Austenización: $1.200{ }^{\circ} \mathrm{C} \times 10 \mathrm{~min}$, primer paso: $1.150{ }^{\circ} \mathrm{C}$, tiempo interpaso: $20-500 \mathrm{~s}, \dot{\varepsilon}=3,63 \mathrm{~s}^{-1}$. (a) $\varepsilon=0,20 ;(b) \varepsilon=0,35$.

Figure 2. Accumulated stress $(\Delta \sigma)$ versus final rolling temperature. Reheating: $1,200{ }^{\circ} \mathrm{C} \times 10 \mathrm{~min}$, first pass: $1,150{ }^{\circ} \mathrm{C}$, interpass time: $20-500 \mathrm{~s}, \dot{\varepsilon}=3,63 \mathrm{~s}^{-1}$. (a) $\varepsilon=0,20$; (b) $\varepsilon=0,35$.

cinética de recristalización. Además, a medida que aumenta $\Delta t$, los precipitados engrosan entre pasos de laminación y pierden su eficacia como obstácu- los del movimiento de los límites de grano austenítico. No obstante, existirá un intervalo de tiempos, cortos pero suficientes, para el comienzo de la precipitación, donde, a mayor $\Delta \mathrm{t}$ mayor será el valor de $T_{\mathrm{nr}}{ }^{[1]}$.

La temperatura de transformación de fase, $A_{\mathrm{r} 3}$, fue determinada por el descenso en la pendiente de la curva MFS durante la zona III. Este leve descenso de pendiente con respecto a la segunda recta de regresión correspondiente a la zona II (originado por la pequeña proporción de ferrita formada) y el hecho de que las regresiones estén trazadas a partir de puntos situados en intervalos de $25^{\circ} \mathrm{C}$ hacen difícil determinar con precisión el valor de $A_{\mathrm{r} 3}$ para varios de los ensayos realizados. De la tabla II se deduce que los valores de $A_{\mathrm{r} 3}$ son, prácticamente, constantes e independientes de $\Delta \mathrm{t}$. En principio, cabría esperar un incremento de $A_{\mathrm{r} 3}$ con $\Delta \sigma$, ya que un aumento de esta última magnitud supone también un incremento en la densidad de dislocaciones y en el área específica de límites de grano, pero hay que tener en cuenta, asimismo, que $T_{\mathrm{nr}}$ decrece con $\Delta \mathrm{t}$, lo que supone que la austenita sufre un mayor número de ciclos de recristalización y, por tanto, su tamaño de grano disminuye, lo que también favorece un incremento de $A_{\mathrm{r} 3}{ }^{[13]}$. Aún más, la velocidad de enfriamiento es un tercer factor que también influye en el valor de la temperatura de transformación, $A_{\mathrm{r} 3}$. En resumen, tiempos de interpaso largos supondrían un incremento en el valor de $A_{\mathrm{r} 3}$ ocasionado por el menor valor de la velocidad de enfriamiento y de $T_{\mathrm{nr}}$, pero por otra parte, los menores valores de $\Delta \sigma$ obtenidos para $\Delta \mathrm{t}$ largos conducirían a un simultáneo descenso en el valor de $A_{\mathrm{r} 3}$. El resultado final ha sido que $A_{\mathrm{r} 3}$ permanece prácticamente

Tabla II. Valores de los parámetros críticos en las simulaciones termomecánicas para diferentes tiempos interpaso $(\Delta t)$ y dos deformaciones $(\varepsilon)$

Table II. Values of critical parameters in thermomechanical simulations for different interpass times $(\Delta t)$ and strains $(\varepsilon)$

\begin{tabular}{|c|c|c|c|c|c|c|}
\hline \multirow[b]{2}{*}{$\Delta t(s)$} & \multicolumn{2}{|c|}{$\varepsilon=0,20$} & \multicolumn{4}{|c|}{$\varepsilon=0,35$} \\
\hline & $\mathrm{T}_{\mathrm{nr}}\left({ }^{\circ} \mathrm{C}\right)$ & $\mathrm{A}_{\mathrm{r} 3}\left({ }^{\circ} \mathrm{C}\right)$ & $\Delta \sigma$ en $\mathrm{A}_{\mathrm{r} 3}(\mathrm{MPa})$ & $\mathrm{T}_{\mathrm{nr}}\left({ }^{\circ} \mathrm{C}\right)$ & $\mathrm{A}_{\mathrm{r} 3}\left({ }^{\circ} \mathrm{C}\right)$ & $\Delta \sigma$ en $\mathrm{A}_{\mathrm{r} 3}(\mathrm{MPa})$ \\
\hline 20 & 897 & 703 & 82,41 & 840 & 698 & 64,33 \\
\hline 30 & 896 & 703 & 83,41 & 819 & 702 & 56,41 \\
\hline 60 & 839 & 700 & 61,01 & 809 & 702 & 48,99 \\
\hline 100 & 819 & 694 & 56,68 & 794 & 698 & 38,24 \\
\hline 200 & 793 & 700 & 44,72 & 744 & 702 & 35,10 \\
\hline 500 & 777 & 698 & 39,08 & 733 & 702 & 30,41 \\
\hline
\end{tabular}


constante para un mismo valor de $\varepsilon$. Cuando se comparan los valores de $A_{r 3}$ correspondientes a las dos diferentes deformaciones empleadas no pueden apreciarse tampoco notables diferencias.

\section{CONCLUSIONES}

- La simulación termomecánica permite conocer las temperaturas críticas de la laminación así como la determinación de la tensión acumulada en la austenita $(\Delta \sigma)$, cuando ésta es deformada a temperaturas comprendidas entre $T_{\mathrm{nr}} \mathrm{y}$ $A_{\mathrm{r} 3}$.

- La determinación de $A_{\mathrm{r} 1}$ mediante el método de la MFS no es posible para aceros con contenido de carbono medio-alto ya que el porcentaje de ferrita es demasiado bajo como para provocar un descenso apreciable en el valor de MFS a bajas temperaturas.

- Para el rango de tiempos interpaso empleado, los valores de $T_{\mathrm{nr}} \mathrm{y}$ de $\Delta \sigma$, medida en $\mathrm{T}=\mathrm{A}_{\mathrm{r} 3}$, decrecen con el tiempo interpaso.

- Asimismo, los valores de $T_{n r}$ y de $\Delta \sigma$ en $A_{r 3}$ son menores cuanto mayor es la deformación aplicada en cada paso.

- $\Delta \sigma$ representa una manera directa y precisa de medir el estado de deformación de la austenita, desde $T_{\mathrm{nr}}$ hasta el comienzo de la transformación $\gamma \rightarrow \alpha\left(T=A_{r 3}\right)$.

- Para obtener microestructuras austeníticas fuertemente deformadas antes de la transformación de fase, que conduzcan a un afino del grano final, es recomendable reducir el tiempo interpaso y la deformación aplicada en los pasos finales de la laminación.

\section{Agradecimientos}

Los autores agradecen la financiación de la beca predoctoral de D. Manuel Gómez, perteneciente al programa I3P del CSIC financiado por el Fondo Social Europeo.

\section{REFERENCIAS}

[1] D.Q. BAI, S.W. YuE, P. Sun y J.J. Jonas, Metall. Trans. A 24 (1993) 2.151-2.159.

[2] F. Boratto, R. Barbosa, S. Yue y J.J. Jonas, Proc. Int. Conf. Physical Metallurgy of Thermomechanical Processing of Steels and Others Metals (THERMEC'88), ISIJ, Tokyo, 1988, pp. 383-390.

[3] M.I. Vega, S.F. Medina, M. Chapa y A. Quispe, ISIJ Int. 39 (1999) 1.304-1.310.

[4] A. Najafi-Zadeh, S. Yue y J.J. JonAs, ISIJ Int. 32 (1992) 213-221.

[5] M. Gómez, S.F. Medina y A. Quispe, IX Cong. Nac. Tratamientos Térmicos y de Superficie, TRATERMAT 2003, I. Garuz, F. Peñalba, M. Cariz y O. Ruano (Eds.), San Sebastián, España, 2003, pp. 53-56.

[6] A. Artigas, M. Páez, Y. Houbaert, A. Monsalve y D. Celentano, Rev. Metal. Madrid 38 (2002) 339-347.

[7] S.F. Medina, M.I. Vega y M. Chapa, Mater. Sci. Technol. 16 (2000) 163-170.

[8] E.T. TURKDOGAN, Iron Steelmaker 3 (1989) 61-75.

[9] A. FAessel, Rev. Métall. Cah. Inf. Tech. 33 (1976) $875-$ 892.

[10] M. Gómez, S.F. MedinA, A. Quispe y P. VAlles, ISIJ Int. 42 (2002) 423-431.

[11] C.M. Sellars, Hot Working and Forming Processes, C.M. Sellars and G.J. Davies (Eds.), Met. Soc., London, 1980, pp. 3-15.

[12] S.F. MEdinA y A. QUisPe, ISIJ Int. 41 (2001) 774-781.

[13] S.F. Medina, M. ChAPA, M. GÓmEZ, A. QuisQue, V. López y B. Fernández, Rev. Metal. Madrid 39 (2003) 408-417. 